\title{
Non-Linear And Cross-Interaction Analyses of SOFA Subscores As Predictive Markers For Sepsis: A Nationwide Retrospective Study
}

Yutaka Umemura ( $\nabla$ plum00022@gmail.com )

Osaka General Medical Center: Osaka Kyuseiki Sogo Iryo Center https://orcid.org/0000-0001-83570313

\section{Kazuma Yamakawa}

Osaka Medical and Pharmaceutical University: Osaka Ika Yakka Daigaku

\section{Shuhei Murao}

Osaka University School of Medicine Graduate School of Medicine: Osaka Daigaku Daigakuin Igakukei Kenkyuka Igakubu

\section{Yumi Mitsuyama}

Osaka General Medical Center: Osaka Kyuseiki Sogo Iryo Center

Hiroshi Ogura

Osaka University School of Medicine Graduate School of Medicine: Osaka Daigaku Daigakuin Igakukei Kenkyuka Igakubu

Satoshi Fujimi

Osaka General Medical Center: Osaka Kyuseiki Sogo Iryo Center

\section{Research}

Keywords: Sepsis, Biomarkers, Mortality, Intensive Care Units, Prognosis, Organ dysfunction.

Posted Date: August 23rd, 2021

DOI: https://doi.org/10.21203/rs.3.rs-824192/v1

License: (c) (i) This work is licensed under a Creative Commons Attribution 4.0 International License. Read Full License 


\section{Abstract}

Background: The Sequential Organ Failure Assessment (SOFA) score is predominantly used to assess the severity of organ dysfunction in sepsis and is definitely proved to be associated with mortality. However, differences in prognostic value between SOFA subscores have not been sufficiently evaluated so far, and detailed evaluation of subscores is required to verify the clinical significance of the SOFA score. The present study aimed to evaluate the non-linear prognostic association of SOFA subscores and the crossinteraction effects on mortality when SOFA subscores were simultaneously increased.

Methods: This retrospective observational study used a part of a large-scale database containing about 30 million patients. Among them, we included all adult patients requiring unplanned hospital admission and were diagnosed as having sepsis by Sepsis-3 criteria from February 2006 to December 2019. A proven/suspected infection was defined as having any of the ICD-10 codes for infection. Associations between the SOFA components and in-hospital mortality were examined using linear and non-linear logistic regression analyses. We also evaluated two-way interaction effects on mortality between an increase of one SOFA subscore and another.

Results: The final study cohort included 38,869 patients with sepsis. Restricted cubic spline analyses showed that an increase in total SOFA score was sharply and linearly associated with increased mortality. However, the prognostic association of SOFA subscores was non-linear and varied widely by biomarker: platelet count showed a J-shaped association, creatinine showed an inverted J-shaped association, and bilirubin showed only a weak association with mortality. The mortality odds ratios of SOFA scores were synergistically increased when another SOFA subscore was higher than 2 points, and the effect modifications were statistically significant in almost all subgroups. Especially in patients with cardiovascular or hepatic subscores of $\geq 2$ points, odds ratios of the other SOFA subscores were remarkably increased (double to triple) compared to those in the whole study population.

Conclusion: Despite the widely varied prognostic associations of SOFA subscores, total SOFA score was sharply and almost linearly associated with increased mortality. Cross-interactions between subscores synergistically enhanced its prognostic associations and might be responsible for the high prognostic accuracy of the total SOFA score.

\section{Introduction}

Sepsis is defined as life-threatening organ dysfunction caused by an overwhelming host response to an infection. ${ }^{1,2}$ Despite the progress in its medical management over the past few decades, sepsis remains an important global health problem, with approximately 11 million sepsis-related deaths reported in 2017..$^{3}$

Development of multiple organ dysfunction is the most important clinical feature during sepsis. The predominant index used to assess organ dysfunction in sepsis is the Sequential Organ Failure 
Assessment (SOFA) score. ${ }^{4}$ A number of studies have provided definite evidence indicating that an increase of the SOFA score in sepsis is associated with an increased probability of mortality. ${ }^{5-7}$

However, the clinical significance of the SOFA subscores has not been sufficiently evaluated so far. For example, weighting in each component of SOFA, such as platelet count, creatinine level, and bilirubin level, was defined to be homogeneous, whereas the pathophysiology of organ dysfunctions is naturally heterogeneous. Furthermore, there is little evidence on the impact on mortality when multiple components in the SOFA subscores are simultaneously increased. The pathophysiology of multiple organ dysfunction cannot be simply considered as an additive combination of each single organ dysfunction. Dysfunctional organs may impact other remote organs through complex, and incompletely understood, biological communication processes. This cross-interaction is so-called organ crosstalk and is considered to accelerate organ dysfunctions. ${ }^{8}$ In other words, certain combinations of SOFA subscores might lead to a synergistically increased mortality in sepsis.

Therefore, detailed prognostic associations and cross-interaction effects of the SOFA subscores need to be elucidated to verify the clinical significance of the SOFA score. To date, some evidence has supported the hypothesis that some biomarkers would non-linearly associate with clinical outcomes. ${ }^{9,10}$ However, the current clinical evidence regarding the non-linear relation between the components of the SOFA subscores and patient prognosis remains limited. Similarly, there is little evidence on the interaction prognostic effects by combinations of the SOFA subscores.

The present study thus aimed to evaluate 1) the detailed, non-linear association between SOFA subscores and mortality and 2) the interaction effects of a simultaneous increase in SOFA subscores on mortality in sepsis using a large, nationwide registry database.

\section{Materials And Methods}

\section{Design and setting}

This retrospective observational study used a part of a large-scale commercial database covering approximately $23 \%$ of acute-care hospitals in Japan that contained about 30 million patients until October 2019 (Medical Data Vision Co., Tokyo, Japan). The database includes data on age, sex, primary diagnoses, concomitant diagnoses, complication diagnoses, procedures, prescriptions, discharge status, and laboratory tests. In this database, the diagnoses are recorded using International Classification of Diseases Tenth Revision (ICD-10) codes. Among the overall patient data registered in the database, this study included patient data from 42 acute-care hospitals having laboratory data.

This study followed the principles of the Declaration of Helsinki. The study protocol was approved by the Institutional Review Board for Clinical Research of Osaka General Medical center (IRB No. S201916015). The board waived the requirement for informed consent because of the anonymous nature of the data and because no information on individual patients, hospitals, or treating physicians was obtained. 


\section{Participants}

We included all patients requiring unplanned hospital admission and were diagnosed as having sepsis from February 1, 2006 to December 31, 2019. In this study, sepsis was defined as having a proven/suspected infection and the development of organ dysfunction (total SOFA score of $\geq 2$ points at the time of admission), according to the Sepsis- 3 criteria. ${ }^{1}$ A proven/suspected infection was defined as having any of the infection-related ICD-10 codes previously proposed by The Institute for Health Metrics and Evaluation (IHME) ${ }^{11}$ in the primary diagnosis or the diagnosis that triggered hospitalization. The detailed set of the ICD-10 codes for the presence of infection is listed in Table S1. We excluded patients who were admitted to hospital with a diagnosis of sepsis more than once during the study period (i.e., had a second or subsequent record of admission).

\section{Data collection}

We collected the following data on baseline patient characteristics: age, sex, height, weight, Charlson Comorbidity Index, ${ }^{12}$ intensive care unit admission, anatomical site of infection, organ dysfunction, use of catecholamines, and laboratory tests including platelet count, white blood cell count, bilirubin, creatinine, prothrombin time, C-reactive protein, blood glucose, lactate dehydrogenase, total protein, albumin, uric acid, blood urea nitrogen, and creatine kinase.

We used previously published ICD-10 coding algorithms for defining Charlson comorbidities. ${ }^{13}$ Organ dysfunction was evaluated by ICD-10 coding algorithms previously published by Angus et al. ${ }^{14}$ and Martin et al. ${ }^{15}$ The presence and severity of organ dysfunction at the time of hospital admission were also evaluated according to the SOFA score.

The renal, hepatic, and coagulation subscores of SOFA were calculated based on the laboratory tests (i.e., creatinine, bilirubin, and platelet count). The cardiovascular, respiratory, and neurological subscores were calculated by a previously published modified method ${ }^{16}$ (Table S2) because sufficient data for the calculation of these subscores by standard methods were not recorded in this study registry. The Japan Coma Scale, used for calculating the neurological subscore instead of the Glasgow Coma Scale, has four main grades (grade 0: alert; grade 1: possible verbal response without any stimulation, not lucid; grade 2: possible eye-opening, verbal and motor response upon stimulation; and grade 3: no eye-opening and coma upon stimulation). The primary outcome measure was all-cause in-hospital mortality.

\section{Statistical analysis}

Descriptive statistics were calculated as group medians with the first and third quartiles for continuous variables, and frequencies with percentages for categorical variables. The differences in patient characteristics, general laboratory test results, and severity of illness scores between survivors and nonsurvivors were evaluated by the Mann-Whitney $\mathrm{U}$ test or chi-square test. 
To evaluate the nonlinear association between mortality and the biomarkers, we constructed restricted cubic spline curves using logistic regression models. The knot values were determined based on Harrell's recommended percentiles, with the knots placed at equally spaced percentiles of the original variable's marginal distribution. ${ }^{17}$ The number of knots in each analysis was determined by the Wald test in such way that the explanatory variables at all sections divided by the knots were significant. ${ }^{18}$

To assess the synergistic increases (interactions) in mortality according to the simultaneous increase in every two SOFA subscores, we evaluated whether the odds ratio of each subscore positive (2 point or more increase) for mortality was significantly increased when another score was also increased above 2 points by logistic regression analyses including two-way interaction terms between the subscores.

Because there were moderate proportions of missing data, we performed sensitivity analyses using a multiple imputation technique to calculate missing values for biomarkers because the probability of missing data for these markers could be assumed not to depend on the unobserved data themselves (missing at random). We created 10 imputations for each missing value using the other available variables and then fit the desired models separately on each of the 10 imputed datasets and combined the results based on the concepts developed by Rubin. ${ }^{19}$

All hypotheses were two-sided, and a $p$ value of $<0.05$ indicated statistical significance. Because of the underpowered nature of the analyses investigating potential heterogeneities of treatment effects, we used a two-sided significance level of $20 \%$ with statistical inferences for the analyses of effect modification. ${ }^{20}$ All statistical analyses were conducted using STATA Data Analysis and Statistical Software version 15.0 (StataCorp LLC, College Station, TX).

\section{Results}

\section{Study population}

During the study period, 186,125 patients were diagnosed as having an infectious disease and required unplanned hospital admission. Among them, we included 41,027 patients who had a total SOFA score of 2 points or more at the time of admission. After excluding 2,158 patients due to a second or subsequent admission to hospital with a diagnosis of sepsis, we finally included 38,869 patients in the final study cohort. Among the study patients, 34,088 patients survived to discharge and 4781 patients died (Fig. 1).

Baseline characteristics, general laboratory test data, and modified SOFA score in the survivors and nonsurvivors are shown in Table 1. Although the non-survivors were significantly older than the survivors (79 vs 84 years old, $p<0.001)$, the sex ratio and Charlson Comorbidity Index were not different between the groups. The distribution of the anatomical site of infection varied significantly between the groups $(p<$ 0.001). The levels of the most recorded laboratory tests, except for that of white blood cells, were significantly different between groups. Similarly, the total modified SOFA score was significantly higher in the non-survivors ( 3 vs 4 , median, $p<0.001$ ). 
Table 1

Patient characteristics

\begin{tabular}{|c|c|c|c|c|}
\hline & Total & Survivors & Non-survivors & \multirow{2}{*}{ Value } \\
\hline & $N=38869$ & $N=34088$ & $N=4781$ & \\
\hline Age, years & $80(69-86)$ & $79(68-86)$ & $84(76-89)$ & $<0.001$ \\
\hline Sex, male & $22904(58.9 \%)$ & $20132(59.1 \%)$ & $2772(58 \%)$ & 0.155 \\
\hline Height $(\mathrm{cm})$ & $158(150-165)$ & $158(150-165)$ & $155(148-163)$ & $<0.001$ \\
\hline Weight (kg) & $53.5(45-63)$ & $\begin{array}{l}54.1(45.8- \\
63.6)\end{array}$ & $47.8(40-56.2)$ & $<0.001$ \\
\hline Charlson Comorbidity Index & $5(2-9)$ & $5(2-10)$ & $5(2-9)$ & $<0.001$ \\
\hline Anatomical site of infection & & & & $<0.001$ \\
\hline Respiratory & $16608(42.7 \%)$ & $13640(40 \%)$ & $2968(62.1 \%)$ & \\
\hline Abdominal & $8777(22.6 \%)$ & $7965(23.4 \%)$ & $812(17 \%)$ & \\
\hline Urinary tract & $6477(16.7 \%)$ & $6046(17.7 \%)$ & $431(9 \%)$ & \\
\hline Bone/soft tissue & $1754(4.5 \%)$ & $1645(4.8 \%)$ & $109(2.3 \%)$ & \\
\hline Central nerve system & $555(1.4 \%)$ & $496(1.5 \%)$ & $59(1.2 \%)$ & \\
\hline Cardiovascular & $420(1.1 \%)$ & $376(1.1 \%)$ & $44(0.9 \%)$ & \\
\hline Other/Unclassifiable & $4278(11 \%)$ & $3920(11.5 \%)$ & $358(7.5 \%)$ & \\
\hline $\begin{array}{l}\text { White blood cell count }\left(10^{3} /\right. \\
\mu \mathrm{L})\end{array}$ & $99(68-138.4)$ & $99(68.5-137.7)$ & $100(66-145)$ & 0.200 \\
\hline C-reactive protein $(\mathrm{mg} / \mathrm{dL})$ & $8.2(2.8-16.1)$ & $7.9(2.6-15.7)$ & $10.2(4.4-18.3)$ & $<0.001$ \\
\hline Platelet count $\left(10^{3} / \mu \mathrm{L}\right)$ & $\begin{array}{l}15.8(11.8- \\
21.9)\end{array}$ & $15.8(12-21.8)$ & $\begin{array}{l}15.9(10.7- \\
23.2)\end{array}$ & $<0.001$ \\
\hline Bilirubin $(\mathrm{mg} / \mathrm{dL})$ & $1(0.6-1.6)$ & $1(0.6-1.7)$ & $0.8(0.5-1.39)$ & $<0.001$ \\
\hline Creatinine $(\mathrm{mg} / \mathrm{dL})$ & $\begin{array}{l}1.06(0.74- \\
1.69)\end{array}$ & $\begin{array}{l}1.04(0.74- \\
1.64)\end{array}$ & $1.21(0.77-2)$ & $<0.001$ \\
\hline Prothrombin time (\%) & $76.3(62-89)$ & $77.5(64-90)$ & $\begin{array}{l}67.8(51.4- \\
82.2)\end{array}$ & $<0.001$ \\
\hline Glucose (mg/dL) & $130(108-165)$ & $129(109-163)$ & $133(104-178)$ & 0.118 \\
\hline Albumin $(\mathrm{g} / \mathrm{dL})$ & $3.3(2.8-3.8)$ & $3.4(2.9-3.8)$ & $2.8(2.3-3.3)$ & $<0.001$ \\
\hline Blood urea nitrogen $(\mathrm{mg} / \mathrm{dL})$ & $23(15.8-36.1)$ & $22(15.2-34.1)$ & $32.4(21.1-50)$ & $<0.001$ \\
\hline Modified SOFA score total & $3(2-4)$ & $3(2-4)$ & $4(3-6)$ & $<0.001$ \\
\hline
\end{tabular}




\begin{tabular}{|cccc|}
\hline Total & Survivors & Non-survivors & Palue \\
\hline $\mathbf{N}=\mathbf{3 8 8 6 9}$ & $\mathrm{N}=\mathbf{3 4 0 8 8}$ & $\mathrm{N}=4781$ & \\
\hline SOFA Sequential Organ Failure Assessment \\
\hline
\end{tabular}

Non-linear associations between in-hospital mortality and the components of the SOFA score are described as restricted cubic spline curves in Fig. 2. An increase in total SOFA score was sharply and almost linearly associated with increased mortality. Although there was little change in predicted mortality as indicated by platelet counts within a range of 150,000 to $500,000 / \mu \mathrm{L}$, the risk of death rose sharply as the platelet counts decreased below a level of approximately $150,000 / \mu \mathrm{L}$. The risk of mortality rose sharply as the levels of creatinine increased from normal range to approximately $3.5 \mathrm{mg} / \mathrm{dL}$, but there was no remarkable increase in mortality according to the increase of creatinine levels when the levels were higher than $5.0 \mathrm{mg} / \mathrm{dL}$. Similarly, an increase in mortality in accordance with the level of bilirubin was observed only when the levels were below approximately $2.0 \mathrm{mg} / \mathrm{dL}$ and was not remarkable when the levels increased above $2.0 \mathrm{mg} / \mathrm{dL}$.

\section{Risk of death according to the increase of SOFA subscores}

The odds ratio for in-hospital death for each point of the SOFA subscores compared to its 0 point is shown in Fig. 3. Increases in the neurological and coagulation subscores were associated with a significant and linear increase in the risk of death. The cardiovascular subscore was also significantly associated with an increase in the risk of death, which skyrocketed when the subscore increased from 3 to 4 points. An increase in the renal subscore was also associated with a significant increase in the risk of death within a range of 1 to 3 points. However, the odds ratio for the subscore of 4 points was relatively lower compared to that for the other subscore points. Similarly, the mortality odds ratio for an hepatic subscore of 4 points was significant but lower compared to those for 1 to 3 points.

\section{Interaction between SOFA subscores}

The cross-interaction effects on mortality between SOFA subscores are summarized as a heat map corresponding to the magnifications of mortality odds ratios of increasing one SOFA subscore when another SOFA subscore was increased (Fig. 4). Each cell contains the following: On the first line: the mortality odds ratios of increasing a SOFA subscore above 2 points in the subgroups when another SOFA subscore is already at 2 points or more; on the second line: the magnifications of odds ratios in the subgroups compared to the whole study patients; and on the third line: the $p$ value for the effect modifications (two-way interactions) on mortality between the increase of one subscore and the increase of another. As a result, the mortality odds ratios of SOFA scores were synergistically increased when another SOFA subscore was higher than 2 points, and the effect modifications were statistically significant in almost all subgroups. Especially in patients with cardiovascular or hepatic subscores of 2 
or more points, the odds ratio of the other SOFA subscores were remarkably increased by double to fourfold compared to those in the whole study population.

\section{Result of multiple imputation}

We performed non-linear regression analyses as sensitivity analyses by imputing the missing values using a multiple imputation technique. As a result, the non-linear associations between mortality and the biomarkers were similar to those without imputations (Figure S1).

\section{Discussion}

\section{Prognostic association in SOFA subscores}

The present study showed that an increase in total SOFA score was almost linearly associated with increased mortality, whereas all three biomarkers of the SOFA subscores were non-linearly associated with the risk of death. The high prognostic value of the total SOFA score has been definitely proven by multiple lines of evidence. ${ }^{21}$ Similarly, the three biomarkers of the SOFA subscores have long been evaluated as biomarkers predictive of mortality. ${ }^{22-24}$ However, there is little evidence regarding the nonlinear prognostic associations of the SOFA subscores, partly because an extremely large sample size is required to construct a non-linear prediction line with high confidence.

Blood coagulation disorders are invariably present in patients with sepsis and play a critical role in the progression of multiple organ dysfunction syndrome. In this study, platelet count showed a J-shaped association with mortality, namely, it was associated with mortality only when decreased below a level of approximately $150,000 / \mu \mathrm{L}$. This finding agreed with that in a previous study evaluating hemostatic biomarkers using multicenter cohort data ${ }^{10}$ and supported the property of the current cutoff point in the SOFA coagulation subscore. Actually, an increase in the coagulation subscore was consistently associated with a significant increase in mortality.

Serum creatinine remains the gold standard for acute kidney injury (AKI), which has long been recognized as an important complication of sepsis independently associated with mortality. ${ }^{24-26}$ In the present study, creatinine showed an inverted J-shaped association, namely, it was associated with mortality when increased from the normal range to approximately $3.5 \mathrm{mg} / \mathrm{dL}$. However, the association with increased mortality was lost when the value increased to above $5.0 \mathrm{mg} / \mathrm{dL}$. The level of creatinine in sepsis-induced AKI at the time of diagnosis is not generally increased above $5.0 \mathrm{mg} / \mathrm{dL}$ but typically ranges between 1.0 to $3.0 \mathrm{mg} / \mathrm{dL} .{ }^{27,28}$ Actually, both the RIFLE and KDIGO criteria for AKI set the creatinine thresholds for worst classification of renal dysfunction at $4.0 \mathrm{mg} / \mathrm{dL} .{ }^{29,30}$ Therefore, the reason for the relatively lower risk in patients with extremely high creatinine levels might be attributable to the increase in the creatinine level above $5.0 \mathrm{mg} / \mathrm{dL}$ possibly not reflecting the acute progression of renal dysfunction by sepsis, but rather reflecting the presence of chronic kidney disease. 
Similarly, the total bilirubin level in the acute phase of sepsis other than in the presence of biliary tract infection has been reported to be associated with increased mortality, but typically, it ranges within a normal to slightly higher level. ${ }^{31,32}$ Extremely high levels of bilirubin (e.g., above $6.0 \mathrm{mg} / \mathrm{dL}$ ) would mainly reflect the presence of biliary tract infection or chronic liver failure, and thus they were not associated with a remarkable increase in the risk of death in the present study when including all anatomical sites of infection.

\section{Interaction of subscores}

Despite the heterogenetic (and partly suboptimal) prognostic values of the subscores, the total SOFA score was sharply and linearly associated with the risk of death. One possible explanation for this high prognostic value of the total SOFA score may be attributable to the interactions of organ dysfunctions, so called organ crosstalk. ${ }^{33}$ For example, the prognostic value of bilirubin (hepatic subscore) was found to be not so high in the present study, but the prognostic values of other subscores were synergistically increased in patients with a higher hepatic subscore. These interactions on mortality would reflect wellknown pathophysiologies in organ crosstalk, such as hepatorenal syndrome, hepatopulmonary syndrome, or hepatic encephalopathy. ${ }^{34,35}$

Also, overwhelming activations of the inflammation and coagulation systems are essential responses for host protection against microbial invasion, but they can also facilitate multiple organ damage through mutual interactions leading to disseminated intravascular coagulation. ${ }^{36}$ These interactions would therefore synergistically enhance the prognostic value of the total SOFA score, even though the prognostic value and cutoff points of some components are suboptimal as single biomarkers.

\section{Limitations}

First, the diagnoses recorded in an administrative claims databases are generally less accurate than those in planned prospective studies because misclassification, underestimation, or overestimation of diagnoses might have occurred. Second, we used a modified SOFA score in the cardiovascular, respiratory, and neurological components because P/F ratio, detailed amounts of catecholamines administered, and the Glasgow Coma Scale score were not recorded in this study registry. Third, this study could not distinguish between pre-existing and newly developed organ dysfunctions because the baseline SOFA score is not recorded in this study registry. To resolve these potential imbalances, our results should be validated by another large-scale clinical study.

\section{Conclusion}

Despite the widely varied prognostic association of SOFA subscores, the total SOFA score was sharply and almost linearly associated with increased mortality. Cross-interactions between subscores synergistically enhanced its prognostic associations and might be responsible for the high prognostic accuracy of the total SOFA score. 


\section{Abbreviations}

AKI

Acute kidney injury; ICD-10:International Classification of Diseases Tenth Revision; IHME:Institute for Health Metrics and Evaluation; SOFA:Sequential Organ Failure Assessment

\section{Declarations}

Conflict of Interest and Source of Funding: None declared.

\section{Ethics approval and consent to participate}

The study protocol was approved by the Institutional Review Board for Clinical Research of Osaka General Medical center (IRB No. S201916015). The board waived the requirement for informed consent because of the anonymous nature of the data and because no information on individual patients, hospitals, or treating physicians was obtained.

\section{Consent for publication}

Not applicable

\section{Availability of data and materials}

The statistical codes and full dataset are available from the corresponding author.

\section{Competing interests}

The authors declare that they have no competing interests.

\section{Funding}

The authors declare that they have no sources of funding to report.

\section{Authors' contributions}

YU conceived and designed this study; contributed to acquisition, shaping data, analyses, and interpretation of the results; and was responsible for drafting, editing, and submission of the manuscript. $\mathrm{KY}$ contributed to the study design; acquisition, analysis, and interpretation of the data; and drafting of the manuscript. SM supported analysis and interpretation of the results. YM, HO, and SF contributed to interpretation of the data and critical appraisal of the manuscript. All of the authors reviewed, discussed, and approved the final manuscript.

\section{Acknowledgements}


The authors thank all the nurses and physicians in the participating institution and all the patients who contributed to this study.

\section{References}

1. Singer M, Deutschman CS, Seymour CW, et al. The Third International Consensus Definitions for Sepsis and Septic Shock (Sepsis-3). JAMA. 2016;315(08):801-10.

2. Shankar-Hari M, Phillips GS, Levy ML, et al. Developing a new definition and assessing new clinical criteria for septic shock: for the Third International Consensus Definitions for Sepsis and Septic Shock (Sepsis-3). JAMA. 2016;315(08):775-87.

3. Rudd KE, Johnson SC, Agesa KM, et al. Global, regional, and national sepsis incidence and mortality, 1990-2017: analysis for the Global Burden of Disease Study. Lancet. 2020;395(10219):200-11.

4. Vincent JL, Moreno R, Takala J, et al. The SOFA (Sepsis-related Organ Failure Assessment) score to describe organ dysfunction/failure. On behalf of the Working Group on Sepsis-Related Problems of the European Society of Intensive Care Medicine. Intensive Care Med. 1996;22(7):707-10.

5. Vincent $J L$, de Mendonça A, Cantraine F, et al. Use of the SOFA score to assess the incidence of organ dysfunction/failure in intensive care units: results of a multicenter, prospective study. Working group on "sepsis-related problems" of the European Society of Intensive Care Medicine. Crit Care Med. 1998;26(11):1793-800.

6. de Grooth HJ, Geenen IL, Girbes AR, Vincent JL, Parienti JJ, Oudemans-van Straaten HM. SOFA and mortality endpoints in randomized controlled trials: a systematic review and meta-regression analysis. Crit Care. 2017;21(1):38.

7. Raith EP, Udy AA, Bailey M, et al. Prognostic accuracy of the SOFA score, SIRS criteria, and qSOFA score for in-hospital mortality among adults with suspected infection admitted to the intensive care unit. JAMA. 2017;317(3):290-300.

8. Pool R, Gomez H, Kellum JA. Mechanisms of organ dysfunction in sepsis. Crit Care Clin. 2018;34(1):63-80.

9. Chen $\mathrm{H}$, Zeng $\mathrm{D}$, Wang Y. Penalized nonlinear mixed effects model to identify biomarkers that predict disease progression. Biometrics. 2017;73(4):1343-54.

10. Matsubara T, Yamakawa K, Umemura Y, et al. Significance of plasma fibrinogen level and antithrombin activity in sepsis: a multicenter cohort study using a cubic spline model. Thromb Res. 2019;181:17-23.

11. Rudd KE, Johnson SC, Agesa KM, et al. Global, regional, and national sepsis incidence and mortality, 1990-2017: Analysis for the Global Burden of Disease Study. Lancet. 2020;395:200-11.

12. Charlson ME, Pompei P, Ales KL, MacKenzie CR. A new method of classifying prognostic comorbidity in longitudinal studies: development and validation. J Chronic Dis. 1987;40:373-83.

13. Quan $\mathrm{H}$, Sundararajan V, Halfon P, et al. Coding algorithms for defining comorbidities in ICD-9-CM and ICD-10 administrative data. Med Care. 2005;43(11):1130-9. 
14. Angus DC, Linde-Zwirble WT, Lidicker J, Clermont G, Carcillo J, Pinsky MR. Epidemiology of severe sepsis in the United States: analysis of incidence, outcome, and associated costs of care. Crit Care Med. 2001;29(7):1303-10.

15. Martin GS, Mannino DM, Eaton S, Moss M. The epidemiology of sepsis in the United States from 1979 through 2000. N Engl J Med. 2003;348(16):1546-54.

16. Yamakawa K, Ohbe H, Taniguchi K, Matsui H, Fushimi K, Yasunaga H. Time trends of the outcomes and treatment options for disseminated intravascular coagulation: a nationwide observational study in Japan. JMA J. 2020;3(4):313-20.

17. Harrell FE Jr. Regression modeling strategies: with applications to linear models, logistic regression, and survival analysis. New York: Springer Verlag; 2001.

18. Judge GG, Griffiths WE, Hill RC, Lütkepohl H, Lee T-C. The theory and practice of econometrics. 2nd ed. New York: John Wiley \& Sons; 1985.

19. Rubin DB. Statistical matching using file concatenation with adjusted weights and multiple imputations. J Bus Econ Stat. 1986;4:87-94.

20. Harell FE Jr. Regression Modeling Strategies. Package 'rms'. Published 2018. Available at: https://cran.rproject.org/web/packages/rms/rms.pdf. Accessed 18 Dec 2018.

21. Ferreira FL, Bota DP, Bross A, Mélot C, Vincent JL. Serial evaluation of the SOFA score to predict outcome in critically ill patients. JAMA. 2001;286(14):1754-8.

22. Venkata C, Kashyap R, Farmer JC, Afessa B. Thrombocytopenia in adult patients with sepsis: incidence, risk factors, and its association with clinical outcome. J Intensive Care. 2013;1(1):9.

23. Patel JJ, Taneja A, Niccum D, Kumar G, Jacobs E, Nanchal R. The association of serum bilirubin levels on the outcomes of severe sepsis. J Intensive Care Med. 2015;30(1):23-9.

24. Brivet FG, Kleinknecht DJ, Loirat $P$, Landais PJ. Acute renal failure in intensive care units-causes, outcome, and prognostic factors of hospital mortality; a prospective, multicenter study. French Study Group on Acute Renal Failure. Crit Care Med. 1996;24:192-8.

25. Chertow GM, Levy EM, Hammermeister KE, Grover F, Daley J. Independent association between acute renal failure and mortality following cardiac surgery. Am J Med. 1998;104:343-8.

26. de Mendonça A, Vincent JL, Suter PM, et al. Acute renal failure in the ICU: risk factors and outcome evaluated by the SOFA score. Intensive Care Med. 2000;26:915-21.

27. Uhel F, Peters-Sengers H, Falahi F, et al. Mortality and host response aberrations associated with transient and persistent acute kidney injury in critically ill patients with sepsis: a prospective cohort study. Intensive Care Med. 2020;46(8):1576-89.

28. Pickkers P, Mehta RL, Murray PT, et al. Effect of human recombinant alkaline phosphatase on 7-day creatinine clearance in patients with sepsis-associated acute kidney injury: a randomized clinical trial. JAMA. 2018;320(19):1998-2009.

29. Bellomo R, Ronco C, Kellum JA, Mehta RL, Palevsky P. Acute Dialysis Quality Initiative workgroup. Acute renal failure - definition, outcome measures, animal models, fluid therapy and information 
technology needs: the Second International Consensus Conference of the Acute Dialysis Quality Initiative (ADQI) Group. Crit Care. 2004;8:R204-12.

30. Kidney Disease. Improving Global Outcomes (KDIGO) Acute Kidney Injury Work Group. KDIGO clinical practice guideline for acute kidney injury. Kidney Int. 2012;2:1-138.

31. Patel JJ, Taneja A, Niccum D, Kumar G, Jacobs E, Nanchal R. The association of serum bilirubin levels on the outcomes of severe sepsis. J Intensive Care Med. 2015;30(1):23-9.

32. Zhai R, Sheu CC, Su L, et al. Serum bilirubin levels on ICU admission are associated with ARDS development and mortality in sepsis. Thorax. 2009;64(9):784-90.

33. Pool R, Gomez H, Kellum JA. Mechanisms of organ dysfunction in sepsis. Crit Care Clin. 2018;34(1):63-80.

34. Simonetto DA, Gines P, Kamath PS. Hepatorenal syndrome: pathophysiology, diagnosis, and management. BMJ. 2020;370:m2687.

35. Kumar P, Rao PN. Hepatopulmonary syndrome. N Engl J Med. 2020;382(10):e14.

36. Iba T, Levy JH, Warkentin TE, et al. Diagnosis and management of sepsis-induced coagulopathy and disseminated intravascular coagulation. J Thromb Haemost. 2019;17(11):1989-94.

\section{Figures}

18,6125 patients diagnosed with infectious disease from February 2006 to December 2019

Criteria for infection

Based on the ICD-10 coding proposed by the Institute for Health Metrics and Evaluation in 2020

41,027 adult patients have a total Sequential Organ Failure Assessment score of $\geq 2$ points at the time of admission

2,158 patients on second or subsequent admission were excluded

38,869 patients with sepsis - Final study cohort

34,088 patients survived to discharge

4,781 patients died

Figure 1 
Patient flow diagram. ICD-10 International Classification of Diseases Tenth Revision
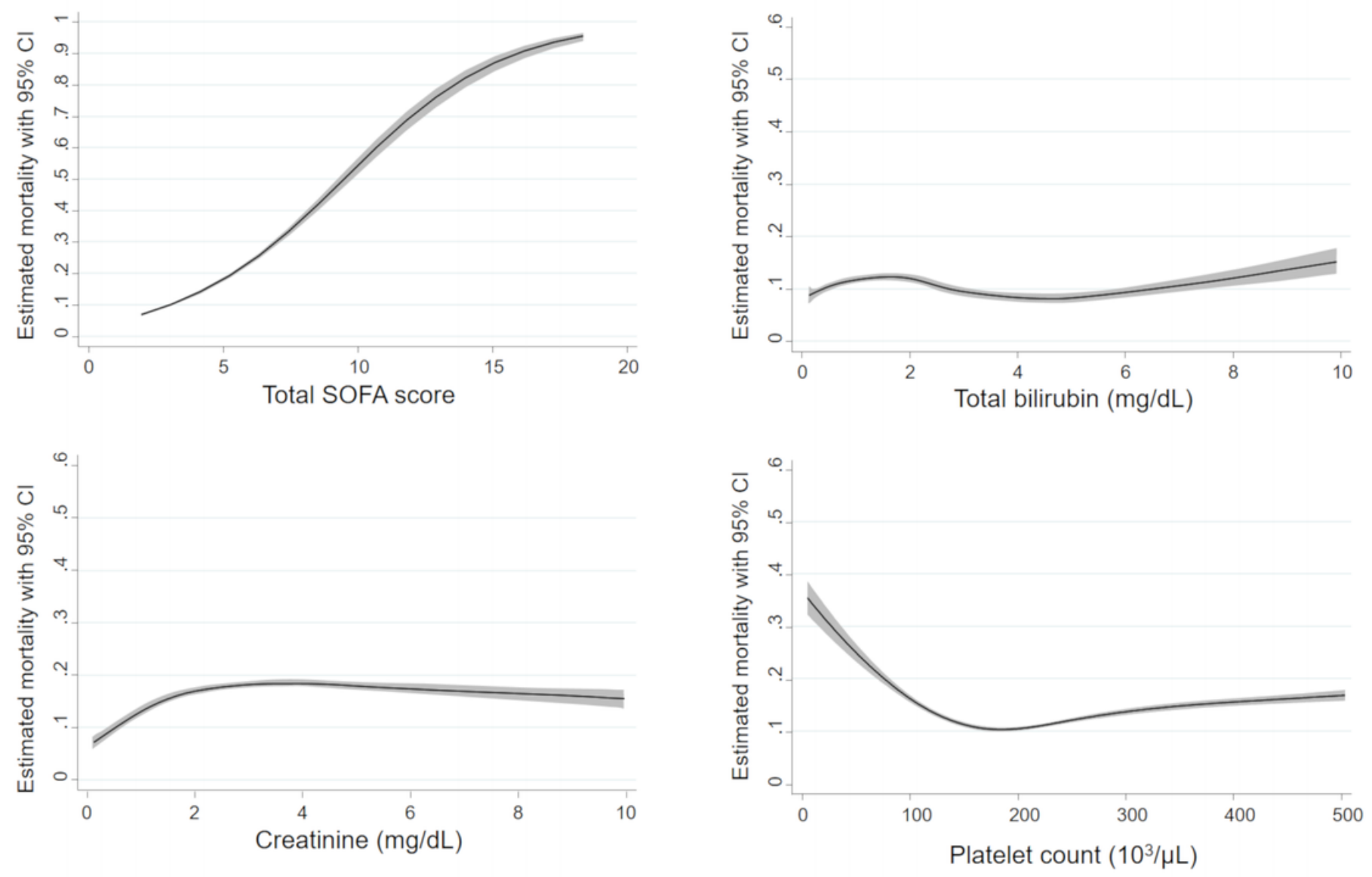

Figure 2

Non-linear association between mortality and the components of the SOFA. The black line represents the fitted line of the association between biomarkers and the estimated mortality risk, and the shaded region represents the $95 \%$ confidence interval. SOFA Sequential Organ Failure Assessment 


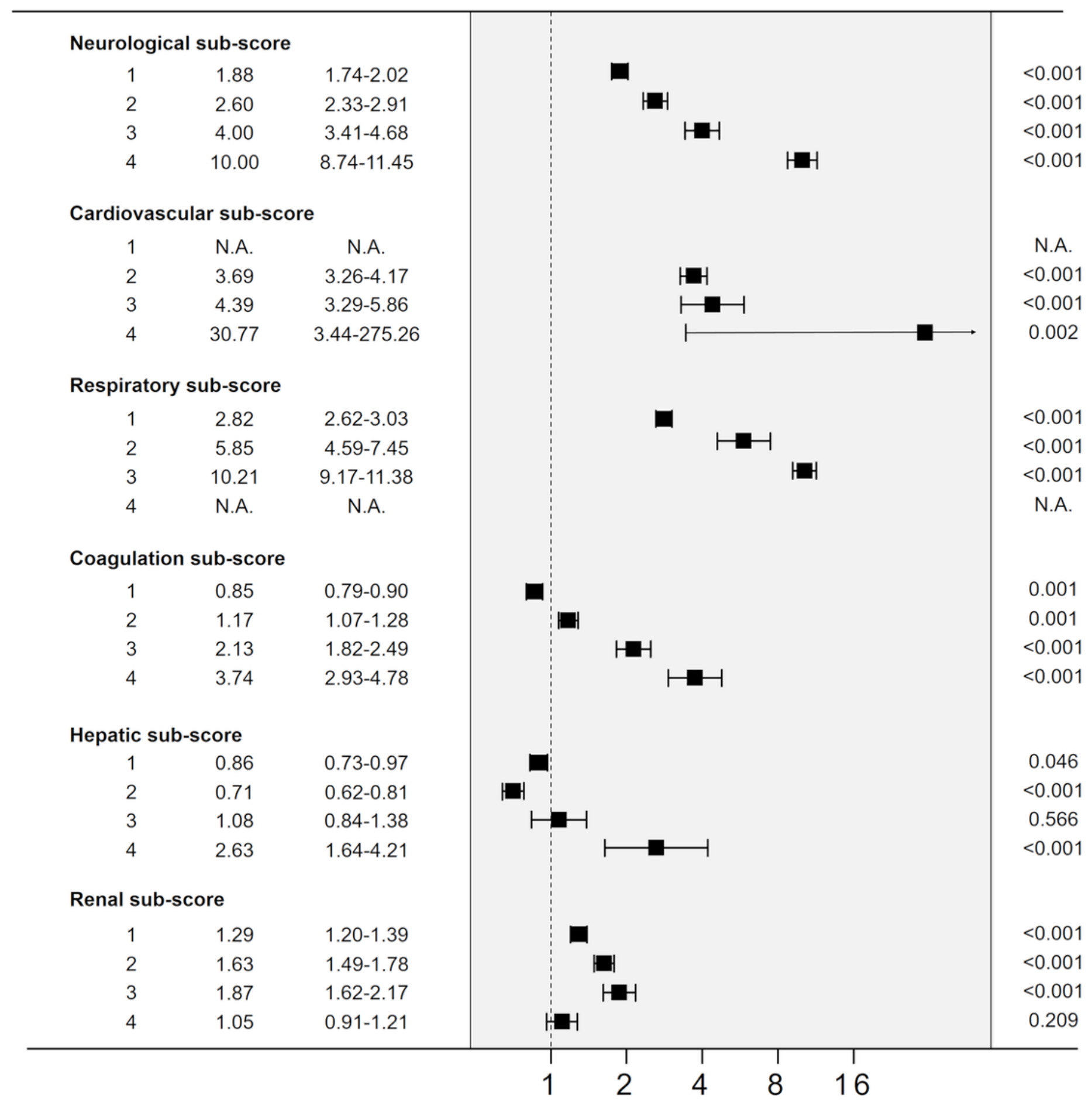

\section{Figure 3}

Mortality odds ratios according to the increase in SOFA subscores, compared to 0 points for each subscore. Cl confidence interval, OR odds ratio, SOFA Sequential Organ Failure Assessment 


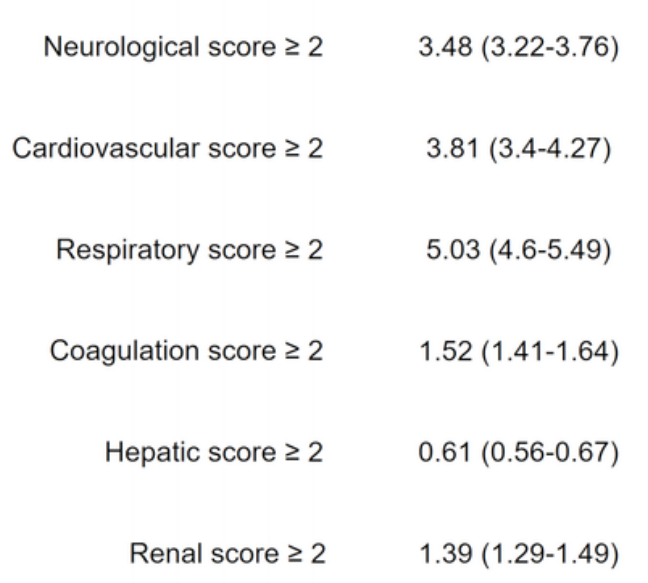

OR for mortality in subgroups,

Magnifications of OR compared to the whole patients, and $\mathrm{p}$ for effect modifications

\begin{tabular}{|c|c|c|c|c|c|c|}
\hline & $\begin{array}{l}\text { OR: } 11.87 \\
(\times 3.41) \\
p<0.001\end{array}$ & $\begin{array}{l}\text { OR: } 3.05 \\
(\times \mathbf{0 . 8 8}) \\
p=0.843\end{array}$ & $\begin{array}{l}\text { OR: } 4.75 \\
(\times 1.36) \\
p=0.003\end{array}$ & $\begin{array}{l}\text { OR: } 7.28 \\
(\times 2.09) \\
p<0.001\end{array}$ & $\begin{array}{l}\text { OR: } 5.81 \\
(\times 1.67) \\
p<0.001\end{array}$ & $\begin{array}{c}\text { of odds ratios } \\
3.5213\end{array}$ \\
\hline $\begin{array}{l}\text { OR: } 9.87 \\
(\times \mathbf{2 . 5 9})\end{array}$ & & $\begin{array}{r}\text { OR: } 4.19 \\
(\times \mathbf{1 . 1 0})\end{array}$ & $\begin{array}{r}\text { OR: } 8.33 \\
(\times \mathbf{2 . 1 9})\end{array}$ & $\begin{array}{r}\text { OR: } 4.49 \\
(\times \mathbf{1 . 1 8})\end{array}$ & $\begin{array}{l}\text { OR: } 9.58 \\
(\times \mathbf{2 . 5 1})\end{array}$ & 3.0719 \\
\hline$p<0.001$ & & $p<0.001$ & $p<0.001$ & $p=0.248$ & $p<0.001$ & 2.8922 \\
\hline $\begin{array}{r}\text { OR: } 4.37 \\
(\times \mathbf{0 . 8 7})\end{array}$ & $\begin{array}{r}\text { OR: } 8.26 \\
(\times 1.64)\end{array}$ & & $\begin{array}{l}\text { OR: } 8.51 \\
(\times \mathbf{1 . 6 9})\end{array}$ & $\begin{array}{c}\text { OR: } 14.75 \\
(\times \mathbf{2 . 9 3})\end{array}$ & $\begin{array}{l}\text { OR: } 6.84 \\
(\times 1.36)\end{array}$ & $\begin{array}{l}2.1724 \\
2.5327\end{array}$ \\
\hline$p=0.843$ & $p<0.001$ & & $p<0.001$ & $p<0.001$ & $p=0.001$ & 2.3529 \\
\hline OR: 2.16 & OR: 3.61 & OR: 2.72 & & OR: 3.17 & OR: 2.15 & 2.1732 \\
\hline$(\times 1.42)$ & $(\times 2.37)$ & $(\times 1.79)$ & & $(\times 2.08)$ & $(\times 1.41)$ & 1.9934 \\
\hline$p=0.003$ & $p<0.001$ & $p<0.001$ & & $p<0.001$ & $p<0.001$ & \\
\hline $\begin{array}{r}\text { OR: } 1.47 \\
(\times \mathbf{2 . 4 0})\end{array}$ & $\begin{array}{r}\text { OR: } 0.74 \\
(\times \mathbf{1 . 2 1})\end{array}$ & $\begin{array}{l}\text { OR: } 2.06 \\
(\times \mathbf{3 . 3 6})\end{array}$ & $\begin{array}{r}\text { OR: } 1.16 \\
(\times \mathbf{1 . 9 0})\end{array}$ & & $\begin{array}{l}\text { OR: } 2.18 \\
(\times 3.57)\end{array}$ & 1.6339 \\
\hline$p<0.001$ & $p=0.248$ & $p<0.001$ & $p<0.001$ & & $p<0.001$ & 1.4542 \\
\hline OR: 2.46 & OR: 4.02 & OR: 1.98 & OR: 1.99 & OR: 4.74 & & 1.2744 \\
\hline$(\times 1.77)$ & $(\times 2.90)$ & $(\times 1.43)$ & $(\times 1.43)$ & $(\times 3.41)$ & & 1.0947 \\
\hline$p<0.001$ & $p<0.001$ & $p=0.001$ & $p<0.001$ & $p<0.001$ & & .91491 \\
\hline
\end{tabular}

\section{Figure 4}

Heat map corresponding to the magnifications of mortality odds ratios in subgroups with each SOFA subscore of 2 points or more. Each cell contains the following: 1) On the first line: the mortality odds ratios of increasing a SOFA subscore above 2 points in the subgroups when another SOFA subscore is already at 2 points or more, 2) on the second line: the magnifications of mortality odds ratios in the subgroups compared to the whole study patients; and 3) on the third line: the p value for the effect modifications (two-way interactions) on mortality between the increase of one subscore and the increase of another. The color key histogram shows the magnifications of mortality odds ratios within each color bar. Cl confidence interval, OR odds ratio, SOFA Sequential Organ Failure Assessment

\section{Supplementary Files}

This is a list of supplementary files associated with this preprint. Click to download.

- Supplementarymaterials.docx 